\title{
RhoC protein stimulates migration of gastric cancer cells through interaction with scaffold protein IQGAP1
}

\author{
YAN WU, YONG-CHANG CHEN, JIAN-RONG SANG and WEN-RONG XU \\ School of Medical Science and Laboratory Medicine, Jiangsu University, Zhenjiang, Jiangsu 212013, P.R. China
}

Received February 28, 2011; Accepted April 18, 2011

DOI: $10.3892 / \mathrm{mmr} .2011 .482$

\begin{abstract}
The scaffold protein IQGAP1 is closely related to certain Rho GTPases. Research has revealed that IQGAP1 acts as an effector of Cdc42 and Rac1 in the regulation of cell activity such as proliferation and migration. However, whether IQGAP1 is associated with RhoC, another important Rho GTPase, is unclear. Previous results from our laboratory indicated that IQGAP1 and RhoC are highly expressed in gastric cancer tissues and cells. This study was designed to investigate the possible interaction between IQGAP1 and $\mathrm{RhoC}$ in the regulation of the migration of cancer cells. The expression of IQGAP1 and RhoC in gastric cancer tissues and cell lines was detected by Western blotting. siRNAs targeting IQGAP1 or RhoC were transfected into gastric cancer cells to knock down the expression of the proteins. Adenoviral constructs encoding full length IQGAP1, the C-terminal fragment of IQGAP1, and the constitutively active RhoC gene were used to infect gastric cancer cells to increase the expression of the proteins. The migratory activity of a gastric cancer cell line was measured by a transwell migration assay. Western blotting revealed that the IQGAP1 and RhoC proteins were highly expressed in gastric cancer tissues and cells. Spearman's rank correlation analysis indicated that the increases in the expression of IQGAP1 and RhoC were closely correlated. The transwell migration assay revealed that both IQGAP1 and RhoC stimulated the migration activity of the gastric cancer cell line AGS. The knockdown of IQGAP1 expression by siRNA blocked the migration-stimulating activity of RhoC, while the knockdown of RhoC expression had no effect on the migration-stimulatory activity of IQGAP1. Co-IP results showed that RhoC and IQGAP1 bound to each other. These results reveal a previously unrecognized interaction between IQGAP1 and RhoC, and demonstrate that IQGAP1 is a downstream effector of $\mathrm{RhoC}$ in the regulation of the migration activity of gastric cancer cells.
\end{abstract}

Correspondence to: Professor Yong-Chang Chen, School of Medical Science and Laboratory Medicine, Jiangsu University, Xue Road, Zhenjiang, Jiangsu 212013, P.R. China

E-mail: ycchen54@ujs.edu.cn

Key words: RhoC, IQGAP1, gastric cancer cell, migration

\section{Introduction}

Ras GTPase-activating-like proteins (IQGAPs) are evolutionary conserved multi-domain proteins that share a similar domain structure and a marked sequence homology. To date, three IQGAPs have been identified in humans. Among them, the best-characterized is IQGAP1, which contains a calponinhomology domain, a tryptophan repeat motif, four centrally located IQ motifs, a C-terminal RasGAP-related domain and a RasGAP-C-terminal domain. As a scaffold protein, IQGAP1 binds multiple proteins and facilitates their interaction. Research has shown that IQGAP1 interacts directly with calmodulin (1), actin (2), E-cadherin $(3,4), \beta$-catenin (5), and the small GTPases Cdc42 (3), Rac1 (6) and Rap1 (7,8). Through its interaction with these proteins, IQGAP1 regulates many cellular functions including cell-cell adhesion, migration (9), cytoskeletal architecture (10), transcription (11) and selected signal transductions.

IQGAP1 has been implicated in tumorigenesis due to the observed overexpression and altered localization of IQGAP1 in neoplasia, particularly at the peripheral regions of more aggressive breast cancer (12), colorectal carcinoma (13) and ovarian adenocarcinomas (14). A lack of IQGAP1 expression is associated with a favorable prognosis in gastric cancer (15). Furthermore, IQGAP1 assists in various signal transductions implicated in cancer. For example, IQGAP1 interacts with MEK, ERK (16) and B-Raf (1) to facilitate their sequential activation and to stimulate MAPK-mediated signaling (17), and promotes proliferation or differentiation (3). IQGAP1 is also capable of directly associating with $\beta$-catenin (18), an oncogenic protein and primary mediator of the Wnt pathway, to cause cell transformation, invasion and migration. Emerging evidence supports a potential role of IQGAP1 in regulating protein traffic (19), cell proliferation and division (20) via the CDC42-mTOR pathway. In another study, it was demonstrated that IQGAP1 facilitated mTOR and Akt interaction and promoted cell proliferation by PI3K/Akt signaling in hepatocellular carcinoma (21). However, although there are adequate data supporting the role of IQGAP1 in tumor progression, what triggers its up-regulation and what key binding partners augment its role in tumorigenicity remain to be determined.

The Rho family of GTPase belongs to the Ras super-family of small $\mathrm{G}$ proteins, which currently comprises more than 20 members. Among them, RhoA, RhoB, and RhoC are 


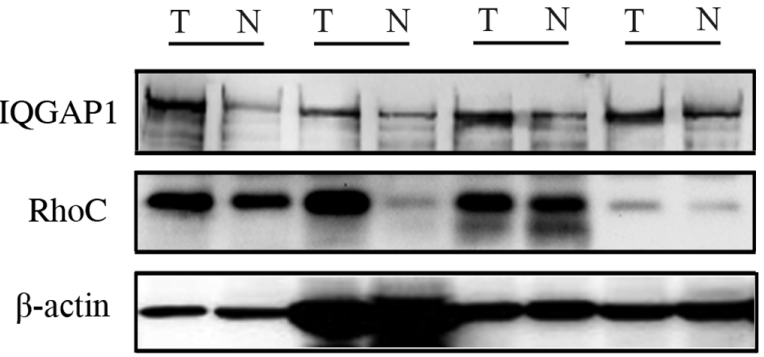

B

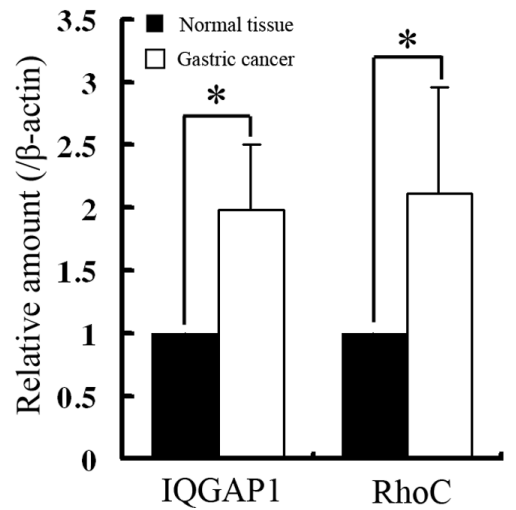

$\mathbf{C}$

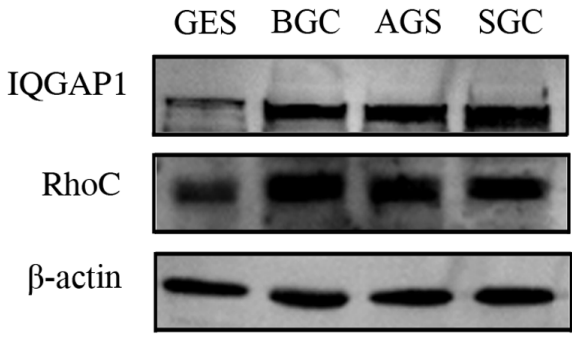

D

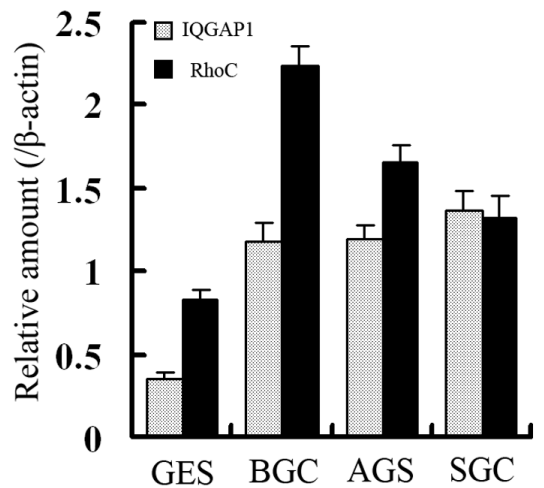

Figure 1. Expression of IQGAP1 and RhoC in gastric cancer tissues and cell lines. (A) Representative results showing alterations in the expression levels of IQGAP1 and RhoC. Proteins were extracted from adjacent normal mucosa and gastric cancer tissues, and Western blotting was used to detect the expression of the target proteins. Lane T, gastric cancer tissues; N, adjacent normal mucosa tissue. (B) Graphical presentation of (A); ${ }^{*}$ p $<0.05$. (C) Western blot analysis of IQGAP1 and RhoC expression levels in GES-1, BGC-823, AGS and SGC-7901 cell lines. (D) Graphical presentation of (C). Values are presented as the means \pm SD obtained from triplicate samples.

Table I. Correlation between RhoC and IQGAP1 expression in 29 gastric cancer patients.

\begin{tabular}{lcrc}
\hline \multirow{2}{*}{$\begin{array}{l}\text { Relative } \\
\text { expression }\end{array}$} & \multicolumn{2}{c}{ No. of cases (\%) } & \\
\cline { 2 - 3 } & RhoC & IQGAP1 & $\begin{array}{c}\text { Statistical } \\
\text { analysis }\end{array}$ \\
\hline $\mathrm{T} / \mathrm{N} \leq 1$ & $7(75.9)$ & $7(75.9)$ & $\mathrm{r}=0.521^{\mathrm{a}}$ \\
$\mathrm{T} / \mathrm{N} \mathrm{1-10}$ & $22(24.1)$ & $22(24.1)$ & $\mathrm{p}=0.004^{\mathrm{a}}$ \\
\hline
\end{tabular}

$\mathrm{T}$, gastric cancer tissue; $\mathrm{N}$, adjacent non-neoplastic mucosa tissue. aSpearman's rank correlation analysis confirmed the statistical significance of the association between RhoC and IQGAP1.

grouped into the Rho subclass. Recent studies have revealed that RhoA and RhoB appear to be associated with the proliferation of cancer cells; nevertheless the overexpression of RhoC enhances the metastasis of cancer cells $(22,23)$. RhoC is frequently overexpressed in a variety of human tumors, and the degree of overexpression correlates with tumor progression and clinical outcome in some cases, such as breast cancer (24), pancreatic cancer (25), colon cancer (26), bladder cancer, hepatocellular carcinoma, non-small cell lung carcinoma (27) and primary gastric tumors (23). In brief, research suggests that RhoC is correlated with tumorigenesis, development and the infiltration metastasis of certain tumor types (28); however, the mechanisms of its action have yet to be elucidated.
IQGAP1 is closely related to some GTPases of the Rho family. For example, both Cdc42 and Rac1, key members of the other two subclasses of the Rho family, interact with IQGAP1 to stimulate the motility of cells. Recently, researchers also observed that Rap1b $(7,8)$ was co-localized with IQGAP1, facilitated the sequential phosphorylation of B-Raf, C-Raf, and ERK1/2, and aided IQGAP1 to form a large signalosome in the perinuclear region. Among the members of the Rho subclass, RhoA (29) has been revealed to have no interaction with IQGAP1. However, it remains to be determined whether RhoC is associated with IQGAP1 or not. Based on our previous observation that the expression of IQGAP1 and RhoC in gastric cancer tissues was markedly higher than that in non-neoplasia mucosa tissues, here we investigated the possible association between IQGAP1 and RhoC.

\section{Materials and methods}

Tissue samples. Gastric cancer tissue specimens $(\mathrm{n}=29)$ were obtained from patients who had undergone surgical resection at the Department of Surgery, Affiliated Hospital of Jiangsu University. None received preoperative chemotherapy or radiotherapy. Fresh specimens were removed from the resected tissues and were immediately frozen at $-80^{\circ} \mathrm{C}$ and stored until use. The corresponding non-neoplasia mucosa tissues, resected at least $5 \mathrm{~cm}$ away from the tumor margin, served as controls. All patients provided written informed consent, and the study protocol and patient information sheets were approved by the Ethics Committee of Jiangsu University. 
A

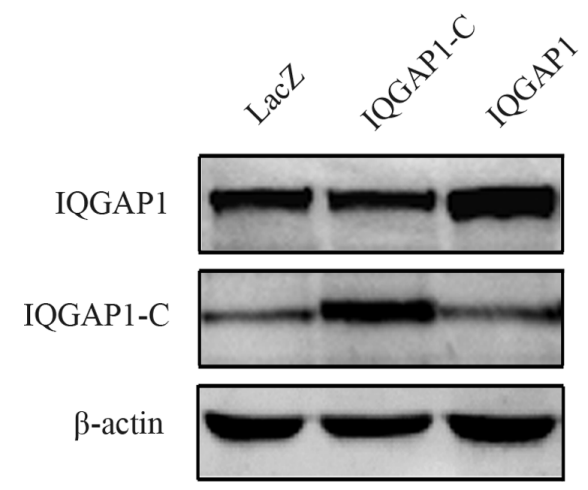

B

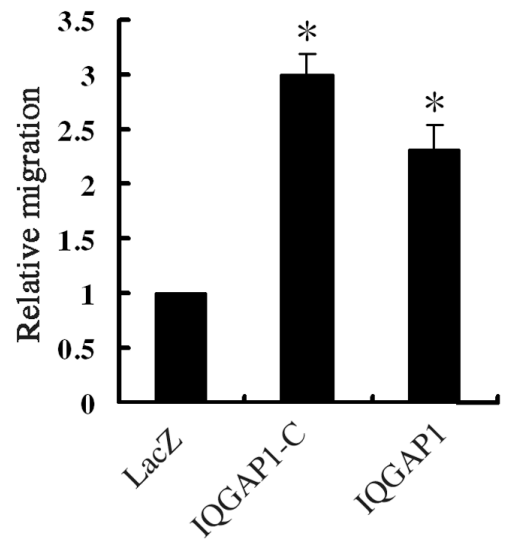

C

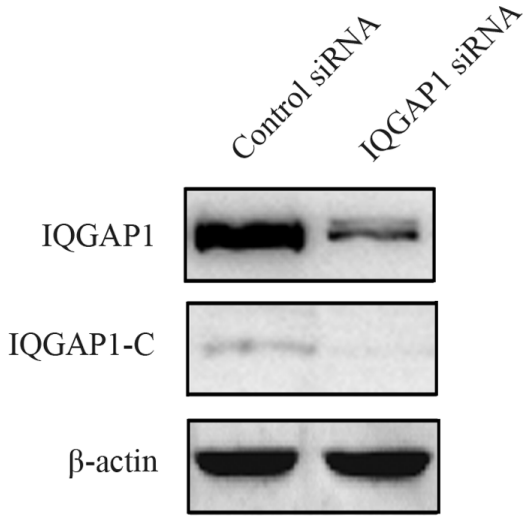

D

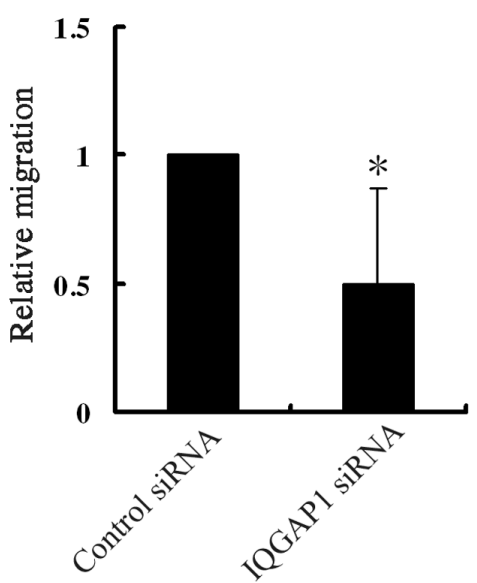

Figure 2. IQGAP1 stimulates the migration of gastric cancer cells. (A) Western blot analysis of IQGAP1 expression in AGS cells infected with pAd-LacZ, pAd-IQGAP1-C or pAd-IQGAP1. (B) The relative migration activity of AGS cells infected with pAd-IQGAP1-C or pAd-IQGAP1 using the transwell migration assay; ${ }^{p}<0.05$ compared to the LacZ group. (C) Protein expression of IQGAP1 in the gastric cancer cell line AGS transfected with IQGAP1 siRNA. (D) Migration assay of AGS cells transfected with IQGAP1 siRNA; " $p<0.05$ compared to control siRNA group. Data are the means \pm SD from three independent experiments, each performed in duplicate.

Reagents, antibodies, siRNA and plasmids. Dulbecco's modified Eagle's medium (DMEM) and fetal bovine serum (FBS) were from Gibco (Grand Island, NY, USA). Lipofectamin $^{\mathrm{TM}} 2000$ cell transfection reagent was from Invitrogen (Carlsbad, CA, USA); mouse anti-RhoC antibody was from Abcam (Cambridge, MA, USA); horseradish peroxidase (HRP)-conjugated secondary antibody was from Rockland Immunochemicals (Gilbertsville, PA, USA); mouse anti-IQGAP1 antibody (against a Glu-Glu tagged C-terminal fragment corresponding to amino acids 863-1657 of human IQGAP1) was from Millipore (Billerica, MA, USA); goat anti- $\beta$-action antibody, and short-interfering RNAs (siRNA) for RhoC and the negative control were purchased from Santa Cruz Biotechnology (Santa Cruz, CA, USA). The siRNA for IQGAP1 was synthesized by Qiagen (Valencia, CA, USA) with a target sequence of 5'-AAGTTCTACGGGAAGTAATTG-3' (5058-5078 bp). Electrochemiluminescence (ECL) reagents were from Millipore; the adenoviral vectors pAd-LacZ (encoding $\beta$-galactosidase), pAd-RhoC-V14 (encoding a constitutive active form of RhoC), pAd-IQGAP1 (encoding the full length IQGAP1) and pAd-IQGAP1-C (encoding the C-terminal fragment of IQGAP1, amino acids 860-1657) were kind gifts from Dr Renate Pilz of the University of California, San Diego, USA; all other reagents used were of analytical grade.
Cell culture, transfection and RNA interference. BGC-823, AGS, GES-1, SGC-7901 and COS-7 cells were cultured in $1 \mathrm{X}$ DMEM containing $10 \% \mathrm{FBS}$ at $37^{\circ} \mathrm{C}$ in a $5 \% \mathrm{CO}_{2}$ atmosphere. The medium was changed every second day and the cells were sub-cultured at confluence. For transfection, the cells were sub-cultured the day before the process. The seeding amount of cells was adjusted to attain a density of $30-50 \%$ confluence on the day of transfection. Transfection of gastric cancer cells with siRNA was performed using Lipofectamin 2000. After 4-6 h, the siRNA/lipid complexes were removed, and the cells were maintained in complete medium for an additional 48-72 h. The inhibition of protein expression was determined by Western blotting.

Western blotting. Cultured cells were washed three times in phosphate-buffered saline (PBS) and lysed with RIPA buffer (50 mM Tris-HCl pH 7.4, 1\% (v/v) Triton X-100, 1 mM EDTA, $1 \mathrm{mM}$ leupeptin, $1 \mathrm{mM}$ PMSF, $10 \mathrm{mM} \mathrm{NaF}, 1 \mathrm{mM} \mathrm{Na} \mathrm{VO}_{4}$ ). Frozen samples of human gastric cancer tissue and normal mucosa tissue were thawed and homogenized in the same buffer. Equal amounts of protein were accumulated with a voltage of $8 \mathrm{~V} / \mathrm{cm}$ and separated with a voltage of $15 \mathrm{~V} / \mathrm{cm}$ on the gel. After electrophoresis, the proteins on the gel were transferred to a polyvinyl difluoride (PVDF) membrane. The membrane was incubated with mouse anti-RhoC antibody 
A

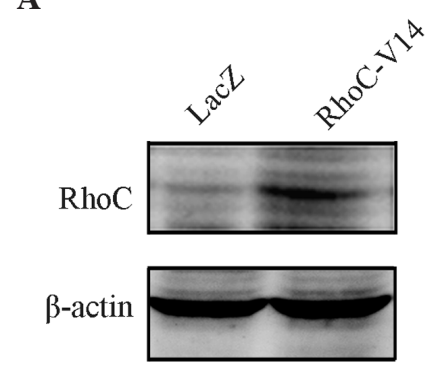

C

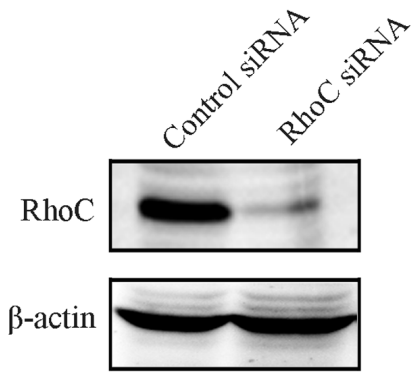

B

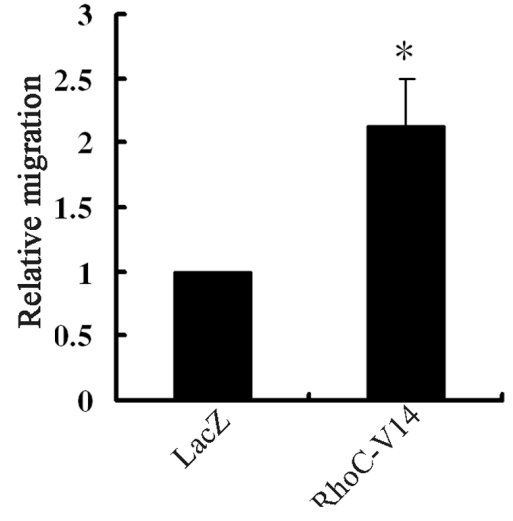

D

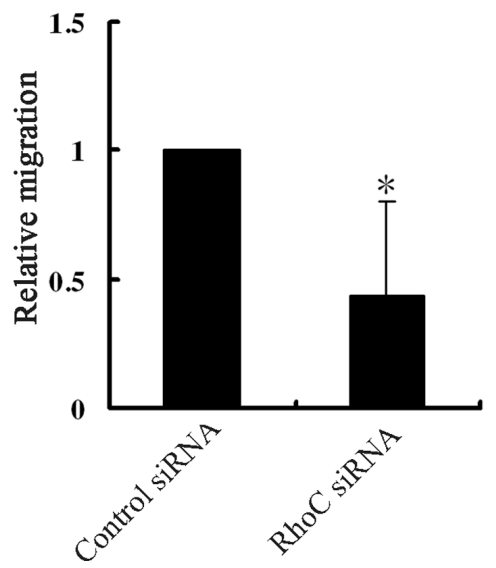

Figure 3. RhoC drives the migration of gastric cancer cells. (A) Western blot analysis of RhoC expression in AGS cells infected with pAd-RhoC-V14. (B) Relative migration activity of AGS cells infected with pAd-RhoC-V14 analyzed by transwell migration chambers; "p<0.05 compared to LacZ group. (C) Protein expression of RhoC in AGS cells transfected with RhoC siRNA. (D) Knock down of RhoC inhibited migration of AGS cells in a transwell migration assay; ${ }^{\mathrm{p}}<0.05$, compared to the control siRNA group. Data are the means \pm SD from three independent experiments, each performed in duplicate.

(diluted 1:500) or mouse anti-IQGAP1 antibody (diluted 1:1000), followed by incubation with the corresponding secondary antibodies. ECL reagents were used to develop the positive bands on the membrane.

Co-immunoprecipitation. To investigate the interaction between IQGAP1 and RhoC, COS-7 cells that had been co-infected for $48 \mathrm{~h}$ with pAd-IQGAP1 and RhoC-V14 or pAd-IQGAP1-C and RhoC-V14 were lysed with RIPA buffer as described. Antibodies against RhoC and IQGAP1 and isotype matched $\mathrm{IgG}$ were used for immunoprecipitation and immunoblotting assay.

Transwell migration assays. Cell migration assays were performed using transwell cell migration chambers (24-well inserts, $8-\mu \mathrm{m}$ pore size; Costar) according to the manufacturer's instructions. AGS cells were transfected with siRNAs targeting IQGAP1 or RhoC for $24 \mathrm{~h}$ and then infected with pAd-RhoC-V14, pAd-IQGAP1-C or pAd-IQGAP1 for an additional $48 \mathrm{~h}$. The cells were serum-starved for $12 \mathrm{~h}$ and then trypsinized, washed, counted, and re-suspended at $2 \times 10^{4}$ cells per well in $300 \mu 1$ of medium containing $0.5 \%$ FBS, and placed in the upper compartment of the chamber. The lower compartment was filled with $500 \mu 1$ of medium containing $10 \% \mathrm{FBS}$. After incubating for $12 \mathrm{~h}$ at $37^{\circ} \mathrm{C}$, the cells remaining in the upper chamber were carefully removed with cotton swabs. Migrated cells on the lower membrane surface were fixed with $4 \%$ paraformaldehyde (PFA) and stained with Giemsa staining solution $\left(1: 20\right.$ in $\left.\mathrm{H}_{2} \mathrm{O}\right)$. In each chamber, cells in five randomly selected high-power (x200) fields were counted and the mean was taken as migration value of this chamber.

Statistical analysis. The data were expressed as the means \pm standard deviation (SD). Statistical significance was performed using two-tailed ANOVA or the Student's t-test with SPSS statistical software. The correlation between IQGAP1 and RhoC was analyzed by Spearman's rank correlation. A p-value $<0.05$ was considered significant.

\section{Results}

Increased expression of IQGAPI and RhoC in gastric cancer tissues and cell lines. In 22 of 29 pairs of samples (75.9\%), the expression levels of IQGAP1 and RhoC were higher in gastric cancer tissues than in non-neoplastic mucosa tissues $(\mathrm{p}<0.05$; Fig. 1A and B; Table I). Correlation analysis by the Spearman's rank test revealed a direct correlation between the expression levels of IQGAP1 and RhoC in gastric cancer tissues ( $\mathrm{p}=0.004$, $\mathrm{r}=0.521$; Table I). In the cell lines, both IQGAP1 and RhoC protein expression levels were increased in the gastric cancer cell lines BGC-823, AGS and SGC-7901 as compared with the human gastric epithelial cell line GES-1 (Fig. 1C and D).

Stimulation of the migration of gastric cancer cells by IQGAPI and RhoC. The effect of IQGAP1 on cell migration was evalu- 
A

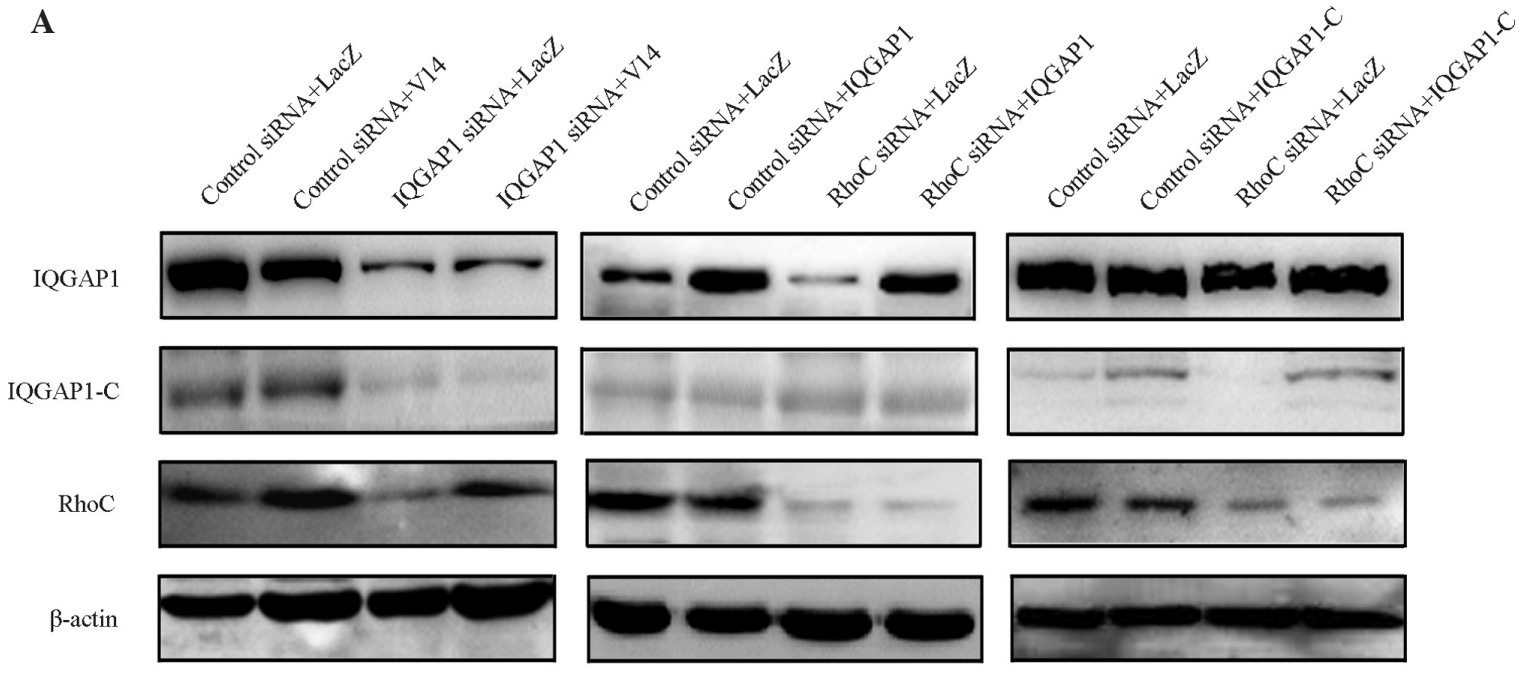

B

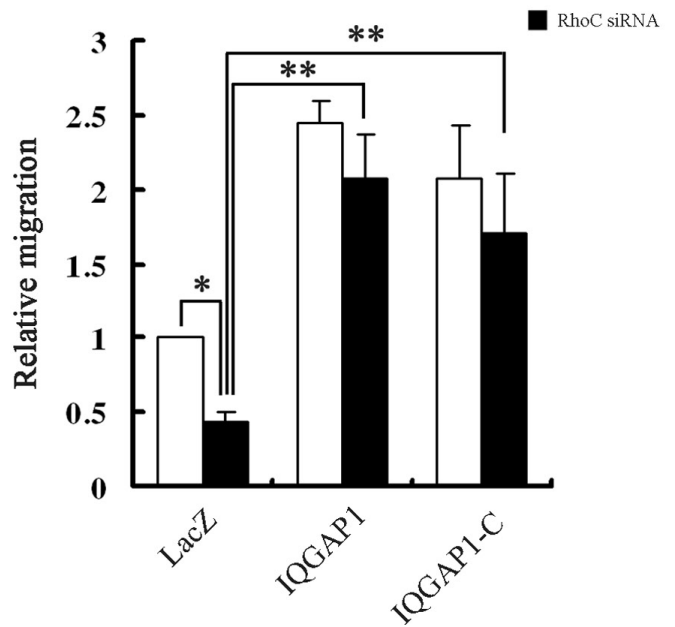

C

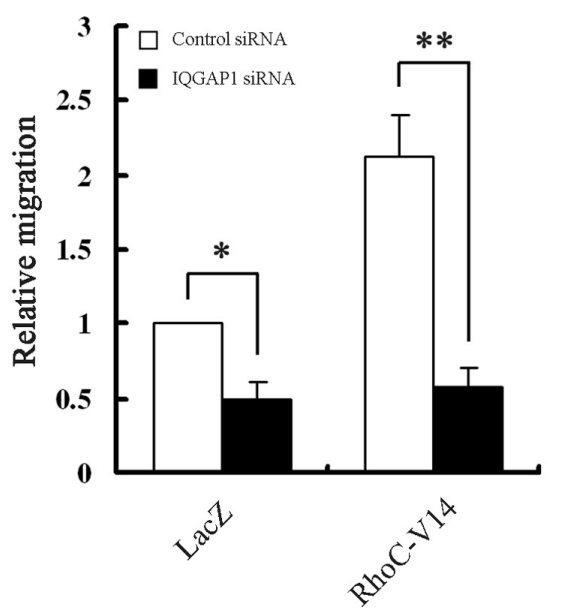

Figure 4. Migration-stimulating effect of RhoC blocked by interference with IQGAP1 expression. (A) Protein expression levels of IQGAP1, IQGAP1-C and RhoC in AGS cells. AGS cells were transiently transfected with IQGAP1 siRNA or RhoC siRNA for $24 \mathrm{~h}$. The transfected cells were subsequently infected with pAd-RhoC-V14, pAd-IQGAP1-C or pAd-IQGAP1 for an additional $48 \mathrm{~h}$ followed by Western blotting. (B) RhoC depletion did not significantly affect IQGAP1 or IQGAP1-C-induced migration in AGS cells. (C) IQGAP1 silencing by siRNA markedly inhibited RhoC-induced cell migration in AGS cells. ${ }^{*} \mathrm{p}<0.05 ;{ }^{* *} \mathrm{p}<0.01$. Data are the means $\pm \mathrm{SD}$ from three independent experiments, each performed in duplicate.

ated by a transwell migration assay. The results revealed that increased expression of full length IQGAP1 (pAd-IQGAP1) or the C-terminal fragment of IQGAP1 (pAd-IQGAP1-C) accelerated cell migration ( $\mathrm{p}<0.05$; Fig. $2 \mathrm{~A}$ and $\mathrm{B})$. By contrast, reduction of endogenous IQGAP1 by siRNA attenuated cell migration by $50 \%$ ( $<<0.05$; Fig. $2 \mathrm{C}$ and D). On the other hand, the results revealed that $\mathrm{RhoC}$ also stimulated the migration of gastric cancer cells. Overexpression of the constitutively active form of RhoC (pAd-RhoC-V14) in AGS cells enhanced migration (Fig. 3A and B). The depletion of RhoC by siRNA inhibited the migration of AGS cells (Fig. 3C and D).

Blockade of RhoC migration-stimulating effect by interference with IQGAPI expression. Since RhoC and IQGAP1 were highly expressed in gastric cancer tissues and cell lines and stimulated the migration of the gastric cancer cell line AGS, we employed several strategies to investigate the possible association between the two proteins. First, AGS cells were transfected with siRNA interfering with the expression of RhoC or IQGAP1, and then infected with adenoviral vector encoding IQGAP1, IQGAP1-C or constitutively active RhoC to determine whether increasing or decreasing one protein would affect the expression of the other. The results revealed that exogenous expression or knockdown of one protein did not significantly affect the expression of the other protein (Fig. 4A). Next, we examined the effect of RhoC knockdown on cell migration stimulated by the full length IQGAP1 or C-terminal fragment of IQGAP1. As shown in Fig. 4B, cell migration was still enhanced in RhoC-siRNA-depletion gastric cancer cells overexpressing the IQGAP1 or IQGAP1 C-terminal fragment $\left({ }^{* *} \mathrm{p}<0.01\right)$. On the other hand, we also tested whether the knock down of IQGAP1 blocked the stimulatory effect of RhoC on cell migration. Fig. 4C showed that the depletion of IQGAP1 by siRNA significantly suppressed the migration enhanced by constitutively active RhoC in gastric cancer cells $\left({ }^{*} \mathrm{p}<0.05,{ }^{* *} \mathrm{p}<0.01\right)$. These data indicated that IQGAP1 served as a downstream effector of RhoC in stimulating the migration of gastric cancer cells, and that the C-terminal fragment of IQGAP1 was the effective region of the protein in association with RhoC. 
A

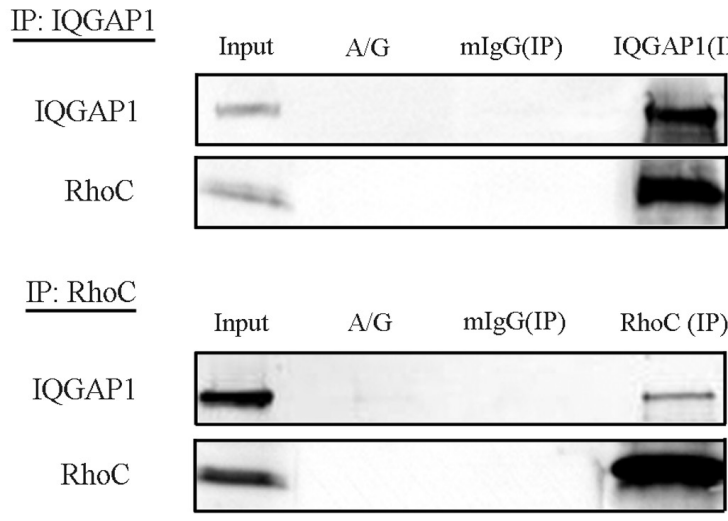

B
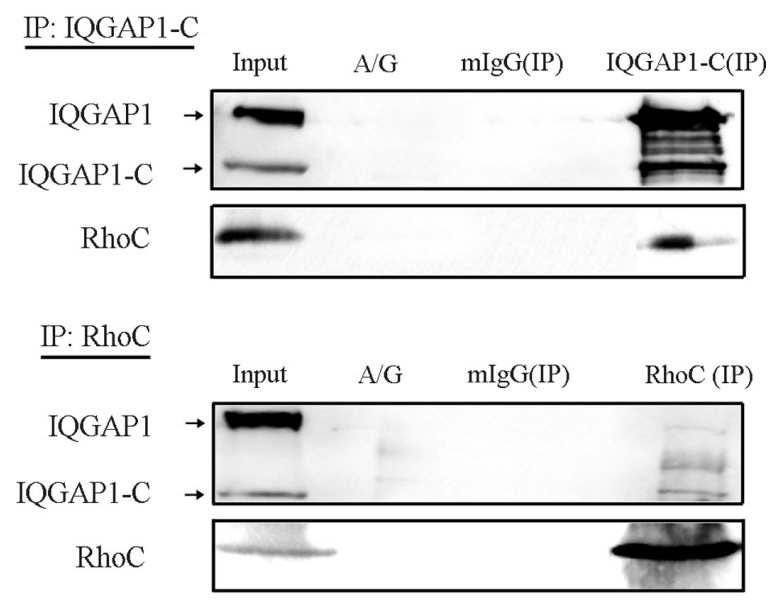

Figure 5. RhoC and IQGAP1 bind to each other. (A) Plates (100 mm) of COS-7 cells were transiently co-infected with pAd-IQGAP1 and pAd-RhoC-V14 for $48 \mathrm{~h}$. The cells were lysed and equal amounts of lysate protein were immunoprecipitated (IP) with anti-RhoC, anti-IQGAP1 antibodies or isotype-matched $\mathrm{IgG}$. Complexes of protein $\mathrm{A} / \mathrm{G}$-Sepharose, antibodies and target proteins were collected through centrifugation and subjected to SDS-PAGE and Western blotting probed with anti-IQGAP1 and anti-RhoC antibodies. Western blotting for the whole cell lysate was used as a protein input control. (B) COS-7 cells were transiently co-infected with pAd-IQGAP1-C and pAd-RhoC-V14 for $48 \mathrm{~h}$. The cells underwent the same Co-IP procedure described above. Data are representative of three independent experiments, with similar results.

RhoC and IQGAPI bind to each other. The co-immunoprecipitation method was used to investigate whether RhoC and IQGAP1 bound to each other. In one group, COS-7 cells were co-infected with pAd-IQGAP1 and pAd-RhoC-V14 for $48 \mathrm{~h}$, and the total cell lysate was immunoprecipitated with anti-RhoC antibody and probed with antibody against IQGAP1. In another group, COS-7 cells were co-infected with pAd-IQGAP1 and RhoC-V14 for $48 \mathrm{~h}$, and the total cell lysate was immunoprecipitated with anti-IQGAP1 antibody and probed with antibody against RhoC. The results revealed that RhoC and IQGAP1 bound to each other (Fig. 5A). To further confirm whether the C-terminal fragment of IQGAP1 was the binding site with RhoC, COS-7 cells were co-infected with pAd-IQGAP1-C and pAd-RhoC-V14 for $48 \mathrm{~h}$, and the total cell lysate was immunoprecipitated with anti-RhoC or anti-IQGAP1 antibodies, respectively. As shown in Fig. 5B, RhoC also bound to the C-terminal fragment of IQGAP1. Together, these data indicate a previously unknown interaction between IQGAP1 and RhoC, and the C-terminal fragment of IQGAP1 as the binding site with RhoC.

\section{Discussion}

Metastasis is the main cause of cancer patient mortality, and the mechanism of metastasis has attracted much research interest. Cell migration is closely related to cancer metastasis, and complex interactions among various proteins and signaling pathways contribute to this process. Rho GTPases have been postulated to participate in regulating migration. For example, Cdc42 and Rac1, two key subclasses of the Rho GTPase family, play important roles in the progression of migration/metastasis (30). RhoC, a key member of the Rho family, is up-regulated in many types of cancer, and the increased expression of $\mathrm{RhoC}$ is correlated with progression and poor prognosis in some types (30-32). The cumulative evidence indicates a significant function of RhoC in the malignant progression of neoplasia, including invasion (33), epithelial-to-mesenchymal transition (EMT) (34), metastasis and proliferation (35). Our results revealed that RhoC was highly expressed in gastric cancer tissues and cell lines, and that the high expression of $\mathrm{RhoC}$ was correlated with the poor differentiation and high invasion of the cancer cells (data not shown). These results are consistent with results from other labs, and further support the involvement of $\mathrm{RhoC}$ in the progression of cancer.

As a scaffold protein, IQGAP1 promotes cell proliferation and motility by binding multiple proteins and regulating their functions. Studies have revealed that, in gastric cancer cells, the overexpression of IQGAP1 enhances cell invasion, while specific knockdown of endogenous IQGAP1 by siRNA significantly reduces cell motility (36). Studies have also indicated that elevated IQGAP1 levels are correlated with the increased expression of certain Rho GTPases, such as active Cdc42 and Rac1 $(37,38)$. In our study, we investigated the expression of IQGAP1 and RhoC in the same clinical samples and found that IQGAP1 and RhoC were highly expressed in cancer tissues. More significantly, our results revealed that changes in the expression of IQGAP1 and RhoC were closely correlated. This indicates that IQGAP1 and RhoC may have a close association/interaction in cancer progression, particularly in regulating migration/metastasis.

Through a transwell migration assay, we investigated the migration-stimulatory effect of IQGAP1 and RhoC on gastric cancer cells. Our results revealed that the induction of IQGAP1 and RhoC expression through infection with adenoviral constructs stimulated the migration of the gastric cancer cell line AGS, and that the siRNA-mediated knockdown of IQGAP1 and RhoC expression inhibited the cell traits. In regulating migration, the knockdown of IQGAP1 expression blocked the migration-stimulating effect of RhoC, while that of RhoC expression did not block the migration-stimulating effect of full length IQGAP1 or the C-terminal fragment of IQGAP1. This indicates that IQGAP1, specifically the C-terminal fragment of IQGAP1, and RhoC were associated with the regulation of migration, and that IQGAP1 may be a downstream effector of RhoC. Through a co-immunoprecipitate assay, we not only observed direct binding between the full length IQGAP1 and RhoC molecules, but also found that it was the C-terminal fragment of IQGAP1 that bound with RhoC, similar to the binding between IQGAP1 and Cdc42 $(20,29)$. Collectively, these data reveal for the first time 
an association between RhoC and IQGAP1, and suggest that IQGAP1 is a downstream effector of RhoC during the regulation of cell migration.

In conclusion, we confirm the effect of IQGAP1 and RhoC on migration in gastric cancer cells, and reveal for the first time the interaction between the two proteins. This will aid in the further understanding of the mechanisms of the migration and metastasis of cancer cells, and suggests novel strategies for cancer therapy.

\section{Acknowledgements}

The authors thank Dr Renate Pilz and Dr Gerry Boss (University of California, San Diego, CA, USA) for providing adenoviral vector pAd-LacZ (encoding $\beta$-galactosidase), pAd-RhoC-V14 (encoding a constitutive active form of RhoC), pAd-IQGAP1 (encoding the full length IQGAP1) and pAd-IQGAP1-C (encoding the C-terminal fragment of IQGAP1, amino acids 860-1657). This research was supported by the National Natural Science Foundation of China (no. 31040002) and an Innovation Grant of Jiangsu University.

\section{References}

1. Ren JG, Li Z and Sacks DB: IQGAP1 integrates Ca2+/calmodulin and B-Raf signaling. J Biol Chem 283: 22972-22982, 2008.

2. Mateer SC, McDaniel AE, Nicolas V, et al: The mechanism for regulation of the F-actin binding activity of IQGAP1 by calcium/ calmodulin. J Biol Chem 277: 12324-12333, 2002.

3. Brown MD and Sacks DB: IQGAP1 in cellular signaling: bridging the GAP. Trends Cell Biol 16: 242-249, 2006.

4. Noritake J, Watanabe T, Wang S, Harada T and Kaibuchi K: Roles of IQGAP1 in E-cadherin-mediated cell-cell adhesion. Tanpakushitsu Kakusan Koso (in Japanese) 51: 648-653, 2006.

5. Lui WY, Mruk DD and Cheng CY: Interactions among IQGAP1, $\mathrm{Cdc} 42$, and the cadherin/catenin protein complex regulate Sertoli-germ cell adherens junction dynamics in the testis. J Cell Physiol 202: 49-66, 2005.

6. Owen D, Campbell LJ, Littlefield K, et al: The IQGAP1-Rac1 and IQGAP1-Cdc42 interactions: interfaces differ between the complexes. J Biol Chem 283: 1692-1704, 2008.

7. Awasthi A, Samarakoon A, Chu H, et al: Rap1b facilitates NK cell functions via IQGAP1-mediated signalosomes. J Exp Med 207: 1923-1938, 2010

8. Jeong HW, Li Z, Brown MD and Sacks DB: IQGAP1 binds Rap1 and modulates its activity. J Biol Chem 282: 20752-20762, 2007.

9. Noritake J, Watanabe T, Sato K, Wang S and Kaibuchi K: IQGAP1: a key regulator of adhesion and migration. J Cell Sci 118: 2085-2092, 2005

10. Smith MJ, Hardy WR, Li GY, et al: The PTB domain of ShcA couples receptor activation to the cytoskeletal regulator IQGAP1. EMBO J 29: 884-896, 2010.

11. Briggs MW, Li ZG and Sacks DB: IQGAP1-mediated stimulation of transcriptional co-activation by beta-catenin is modulated by calmodulin. J Biol Chem 277: 7453-7465, 2002.

12. Jadeski L, Mataraza JM, Jeong HW, Li Z and Sacks DB: IQGAP1 stimulates proliferation and enhances tumorigenesis of human breast epithelial cells. J Biol Chem 283: 1008-1017, 2008.

13. Hayashi H, Nabeshima K, Aoki M, et al: Overexpression of IQGAP1 in advanced colorectal cancer correlates with poor prognosis-critical role in tumor invasion. Int $\mathrm{J}$ Cancer 126: 2563-2574, 2010

14. Dong P, Nabeshima K, Nishimura $\mathrm{N}$, et al: Overexpression and diffuse expression pattern of IQGAP1 at invasion fronts are independent prognostic parameters in ovarian carcinomas. Cancer Lett 243: 120-127, 2006.
15. Walch A, Seidl S, Hermannstadter C, et al: Combined analysis of Rac1, IQGAP1, Tiam1 and E-cadherin expression in gastric cancer. Mod Pathol 21: 544-552, 2008

16. Roy M, Li ZG and Sacks DB: IQGAP1 binds ERK2 and modulates its activity. J Biol Chem 279: 17329-17337, 2004.

17. Roy M, Li Z and Sacks DB: IQGAP1 is a scaffold for mitogenactivated protein kinase signaling. Mol Cell Biol 25: 7940-7952, 2005.

18. Shimao $\mathrm{Y}$, Nabeshima K, Inoue $\mathrm{T}$ and Koono M: Complex formation of IQGAP1 with E-cadherin/catenin during cohort migration of carcinoma cells. Its possible association with localized release from cell-cell adhesion. Virchows Arch 441: 124-132, 2002

19. Osman M: An emerging role for IQGAP1 in regulating protein traffic. ScientificWorldJournal 10: 944-953, 2010.

20. Wang JB, Sonn R, Tekletsadik YK, Samorodnitsky D and Osman MA: IQGAP1 regulates cell proliferation through a novel CDC42-mTOR pathway. J Cell Sci 122: 2024-2033, 2009.

21. Chen F, Zhu HH, Zhou LF, Wu SS, Wang J and Chen Z: IQGAP1 is overexpressed in hepatocellular carcinoma and promotes cell proliferation by Akt activation. Exp Mol Med 42: 477-483, 2010.

22. Wheeler AP and Ridley AJ: Why three Rho proteins? RhoA, RhoB, RhoC, and cell motility. Exp Cell Res 301: 43-49, 2004.

23. Liu N, Zhang G, Bi F, et al: RhoC is essential for the metastasis of gastric cancer. J Mol Med 85: 1149-1156, 2007.

24. Wu M, Wu ZF, Rosenthal DT, Rhee EM and Merajver SD: Characterization of the roles of RHOC and RHOA GTPases in invasion, motility, and matrix adhesion in inflammatory and aggressive breast cancers. Cancer 116: 2768-2782, 2010.

25. Dietrich KA, Schwarz R, Liska M, et al: Specific induction of migration and invasion of pancreatic carcinoma cells by RhoC, which differs from RhoA in its localisation and activity. Biol Chem 390: 1063-1077, 2009.

26. Espejo R, Rengifo-Cam W, Schaller MD, Evers BM and Sastry SK: PTP-PEST controls motility, adherens junction assembly, and Rho GTPase activity in colon cancer cells. Am J Physiol Cell Physiol 299: 454-463, 2010.

27. Han Y, Wu Y, Gao S, et al: Expression and clinical significance of RhoC and RhoGDIa in non-small cell lung cancer. Zhongguo Fei Ai Za Zhi (in Chinese) 11: 391-397, 2008.

28. Ellenbroek SIJ and Collard JG: Rho GTPases: functions and association with cancer. Clin Exp Metastasis 24: 657-672, 2007.

29. Hart MJ, Callow MG, Souza B and Polakis P: IQGAP1, a calmodulin-binding protein with a rasGAP-related domain, is a potential effector for cdc42Hs. EMBO J 15: 2997-3005, 1996.

30. Vega FM and Ridley AJ: Rho GTPases in cancer cell biology. FEBS Lett 582: 2093-2101, 2008.

31. Sahai E and Marshall CJ: RHO-GTPases and cancer. Nature Reviews Cancer 2: 133-142, 2002.

32. Zhao Y, Zong ZH and Xu HM: RhoC expression level is correlated with the clinicopathological characteristics of ovarian cancer and the expression levels of ROCK-I, VEGF, and MMP9. Gynecol Oncol 116: 563-571, 2010.

33. Wu M, Wu ZF, Rosenthal DT, Rhee EM and Merajver SD: Characterization of the roles of RHOC and RHOA GTPases in invasion, motility, and matrix adhesion in inflammatory and aggressive breast cancers. Cancer 116 (Suppl 11): 2768-2782, 2010.

34. Bellovin DI, Simpson KJ, Danilov T, et al: Reciprocal regulation of RhoA and RhoC characterizes the EMT and identifies RhoC as a prognostic marker of colon carcinoma. Oncogene 25: 6959-6967, 2006.

35. Faried A, Faried LS, Kimura H, et al: RhoA and RhoC proteins promote both cell proliferation and cell invasion of human oesophageal squamous cell carcinoma cell lines in vitro and in vivo. Eur J Cancer 42: 1455-1465, 2006.

36. Johnson M, Sharma M and Henderson BR: IQGAP1 regulation and roles in cancer. Cell Signal 21: 1471-1478, 2009.

37. Mataraza JM, Briggs MW, Li ZG, Entwistle A, Ridley AJ and Sacks DB: IQGAP1 promotes cell motility and invasion. J Biol Chem 278: 41237-41245, 2003.

38. Bielak-Zmijewska A, Kolano A, Szczepanska K, Maleszewski M and Borsuk E: Cdc42 protein acts upstream of IQGAP1 and regulates cytokinesis in mouse oocytes and embryos. Dev Biol 322: $21-32,2008$ 